Algebraic $8 \mathcal{G}$ Geometric $\mathcal{T}_{\text {opology }}$

Volume 4 (2004) 595-602

Published: 16 August 2004

ATG

\title{
Commutators and squares in free groups
}

\author{
SUCHARIT SARKAR
}

\begin{abstract}
Let $\mathbb{F}_{2}$ be the free group generated by $x$ and $y$. In this article, we prove that the commutator of $x^{m}$ and $y^{n}$ is a product of two squares if and only if $m n$ is even. We also show using topological methods that there are infinitely many obstructions for an element in $\mathbb{F}_{2}$ to be a product of two squares.
\end{abstract}

AMS Classification 20F12; 57M07

Keywords Commutators, free groups, products of commutators

\section{Introduction}

In any group $G$, the commutator of two elements $g$ and $h$ is a product of three squares, namely,

$$
g h g^{-1} h^{-1}=g^{2}\left(g^{-1} h\right)^{2} h^{-2} .
$$

Let $[g, h]$ denote the commutator of $g$ and $h$. It is natural to ask whether $[g, h]$ can be written as a product two squares. Since the subgroup generated by $g$ and $h$ in $G$ is a quotient of the free group on two generators, the answer would be in the affirmative if we knew that the commutator of the generators of the free group on two generators can be written as a product of two squares. However, if $\mathbb{F}_{2}$ is the free group on two generators $x$ and $y$, a theorem of Lyndon and Newman [2] states that the commutator $[x, y]$ is not a product of two squares. Here, we give the following generalisation of their theorem. Further, the method of our proof extends to give infinitely many obstructions to an element being the product of two squares.

Theorem 1.1 $\left[x^{m}, y^{n}\right]$ is a product of two squares in $\mathbb{F}_{2}=\langle x, y\rangle$ if and only if $m n$ is even.

In the case when $m=n=1$ this gives the theorem of Lyndon and Newman. Our methods also give a proof of the following theorem of Akhavan-Malayeri [1]. 
Theorem 1.2 (Akhavan-Malayeri) $[x, y]^{2 n+1}$ is not a product of two squares in $\mathbb{F}_{2}=\langle x, y\rangle$.

We first reformulate the question in terms of products of conjugate elements.

Lemma 1.3 An element $g \in \mathbb{F}_{2}$ can be expressed as a product $g=a^{2} b^{2}$ of two squares if and only if $g$ can be expressed as a product $g=c\left(d^{-1} c d\right)$ of two conjugate elements.

Proof This follows immediately from the relation $a^{2} b^{2}=(a b)\left(b^{-1}(a b) b\right)$.

The following statement underlines the importance of the previous lemma.

Lemma 1.4 Suppose $g \in\left[\mathbb{F}_{2}, \mathbb{F}_{2}\right]$ is of the form $g=a b$ with $a$ and $b$ conjugate in $\mathbb{F}_{2}$. Then $a, b \in\left[\mathbb{F}_{2}, \mathbb{F}_{2}\right]$.

Proof Let $g \mapsto \bar{g}$ be the abelianisation homomorphism $\mathbb{F}_{2} \rightarrow \mathbb{Z}^{2}$. Then as $g \in\left[\mathbb{F}_{2}, \mathbb{F}_{2}\right], 0=\bar{g}=\bar{a}+\bar{b}=2 \bar{a}$ where the last equality holds as $a$ and $b$ are conjugate. Thus, $\bar{a}=\bar{b}=0$ which implies that $a, b \in\left[\mathbb{F}_{2}, \mathbb{F}_{2}\right]$.

The heart of our proof lies in constructing a group homomorphism $\varphi:\left[\mathbb{F}_{2}, \mathbb{F}_{2}\right] \rightarrow$ $\mathbb{Z}$ which is invariant under conjugacy action of $\mathbb{F}_{2}$, i.e., $\varphi(a)=\varphi\left(g^{-1} a g\right)$ for all $a \in\left[\mathbb{F}_{2}, \mathbb{F}_{2}\right]$ and $g \in \mathbb{F}_{2}$. The construction of our homomorphism is topological.

If an element $g \in\left[\mathbb{F}_{2}, \mathbb{F}_{2}\right]$ is a product of two squares, then we shall see that $\varphi(g)$ is even. This gives a criterion to decide whether $g$ is the product of two squares. The theorem of Lyndon and Newman follows from this. We shall extend this to stronger criteria for an element in $\left[\mathbb{F}_{2}, \mathbb{F}_{2}\right]$ to be a product of two squares.

\section{The homomorphism $\varphi$}

We construct a homomorphism $\varphi:\left[\mathbb{F}_{2}, \mathbb{F}_{2}\right] \rightarrow \mathbb{Z}$ which is invariant under the conjugacy action of $\mathbb{F}_{2}$. The theorem of Lyndon and Newman follows from the properties of this homomorphism.

Let $K$ be the wedge of two circles. Then $\pi_{1}(K)=\mathbb{F}_{2}$. Let $\tilde{K}$ be the universal abelian cover of $K$. This is the cover corresponding to the subgroup $\left[\mathbb{F}_{2}, \mathbb{F}_{2}\right]$ of $\mathbb{F}_{2}$. We can identify $\tilde{K}$ with a subcomplex of $\mathbb{R}^{2}$ whose vertices are $\mathbb{Z}^{2}$ and edges join $(i, j)$ either to $(i+1, j)$ or to $(i, j+1)$. 
Let $X$ and $Y$ denote the edges from $(0,0)$ to $(1,0)$ and $(0,1)$ respectively. Under the group $\mathbb{Z}^{2}$ of deck transformations each edge is the image of $X$ or $Y$. Denoting the group of deck transformations multiplicatively and taking $x$ and $y$ to be the generators, we see that all edges are of the form $x^{i} y^{j} X$ or $x^{i} y^{j} Y$ with $i, j \in \mathbb{Z}$.

Consider the simplicial homology of $\tilde{K}$. We shall first define a homomorphism $\theta: Z_{1}(\tilde{K}) \rightarrow \mathbb{Z}$ from the 1 -cycles to the integers which is invariant under deck transformations. The homomorphism $\varphi$ will be defined in terms of this.

Observe that, using the above notation, the simplicial chains of $\tilde{K}$ are of the form $\alpha=P_{\alpha}(x, y) X+Q_{\alpha}(x, y) Y$ with $P_{\alpha}(x, y)$ and $Q_{\alpha}(x, y)$ Laurent polynomials. Let $f_{\alpha}(y)=P_{\alpha}(1, y)$. The action by a deck transformation $x^{k} y^{l}$ takes $\alpha=P_{\alpha}(x, y) X+Q_{\alpha}(x, y) Y$ to $x^{k} y^{l} \alpha=x^{k} y^{l} P_{\alpha}(x, y) X+x^{k} y^{l} Q_{\alpha}(x, y) Y$.

Further, for a cycle $\alpha$, it is easy to see that $f_{\alpha}(1)=P_{\alpha}(1,1)=0$. We define the homomorphism $\theta$ by

$$
\theta(\alpha)=f_{\alpha}^{\prime}(1)
$$

Lemma 2.1 $\theta: Z_{1}(\tilde{K})=H_{1}(\tilde{K}) \rightarrow \mathbb{Z}$ is invariant under the group of deck transformations.

Proof It suffices to show that $\theta(x \alpha)=\theta(\alpha)=\theta(y \alpha)$. The first equality is obvious as $f_{x \alpha}=f_{\alpha}$. The second follows as

$$
f_{y \alpha}^{\prime}(1)=\left(y f_{\alpha}\right)^{\prime}(1)=f_{\alpha}(1)+f_{\alpha}^{\prime}(1)=f_{\alpha}^{\prime}(1) .
$$

Here we used the fact that for a cycle $f_{\alpha}(1)=0$.

As $\tilde{K}$ is a 1-complex, this can be viewed as a homomorphism, also denoted $\theta$, from $H_{1}(\tilde{K})$ to $\mathbb{Z}$. Using this, we define $\varphi:\left[\mathbb{F}_{2}, \mathbb{F}_{2}\right] \rightarrow \mathbb{Z}$. Namely, given a curve $\gamma$ in $K$ representing an element $g \in\left[\mathbb{F}_{2}, \mathbb{F}_{2}\right]$, take a lift $\tilde{\gamma}$ of $\gamma$ to $\tilde{K}$. This represents an element in homology, and we let $\varphi(g)=\theta(\tilde{\gamma})$. This is independent of the lift chosen as different lifts are related by deck transformation, and $\theta$ is invariant under deck transformations.

We need some properties of $\varphi$.

Lemma 2.2 $\varphi(g)=\varphi\left(h g h^{-1}\right)$ for all $g \in\left[\mathbb{F}_{2}, \mathbb{F}_{2}\right]$ and $h \in \mathbb{F}_{2}$.

Proof The elements of $g$ and $h g h^{-1}$ of $\left[\mathbb{F}_{2}, \mathbb{F}_{2}\right]$ can be represented by curves that have lifts in $\tilde{K}$ that differ by deck transformations. As deck transformations leave $\theta$ invariant, $\varphi(g)=\varphi\left(h g h^{-1}\right)$. 
Lemma 2.3 Suppose $g \in\left[\mathbb{F}_{2}, \mathbb{F}_{2}\right]$ is a product of two squares. Then $\varphi(g)$ is even.

Proof By Lemma 1.3 we can write $g=h k$ with $h$ and $k$ elements of $\left[\mathbb{F}_{2}, \mathbb{F}_{2}\right]$ that are conjugate in $\mathbb{F}_{2}$. We have $\varphi(g)=\varphi(h)+\varphi(k)=2 \varphi(h)$ as $\varphi$ is invariant under conjugation.

Consider the lift to $\tilde{K}$ of a curve $\gamma$ representing $[x, y]$ in $K$. This lift gives the cycle $\alpha=(1-y) X+(x-1) Y$ and, hence $\varphi([x, y])=f_{\alpha}^{\prime}(1)=-1$. Thus, $\varphi$ is non-trivial. Moreover, by the above lemma $[x, y]$ is not a product of two squares.

We now prove our extension of the result of Lyndon and Newman, one half of which is an extension of the above argument.

Proof of Theorem 1.1 Take a curve $\gamma$ representing $\left[x^{m}, y^{n}\right]$. We see that the lift of this starting at the origin gives the chain

$$
\alpha=\left(1+x+\ldots x^{m-1}\right)\left(1-y^{n}\right) X+\left(1+y+\cdots+y^{n-1}\right)\left(x^{m}-1\right) Y
$$

and hence $\varphi\left(\left[x^{m}, y^{n}\right]\right)=-m n$. Hence if $\left[x^{m}, y^{n}\right]$ is the product of two squares, $m n$ is even.

Conversely, if $m n$ is even, we assume without loss of generality that $m$ is even. Then $\left[x^{m}, y^{n}\right]=\left(x^{\frac{m}{2}}\right)^{2}\left(y^{n} x^{\frac{-m}{2}} y^{-n}\right)^{2}$.

The same methods also yield a proof of Theorem 1.2 of Akhavan-Malayeri. We thank the referee for pointing this out.

Proof of Theorem 1.2 By the above, $\varphi\left([x, y]^{2 n+1}\right)=-(2 n+1)$ as $\varphi$ is a homomorphism. The result follows.

We have constructed a homomorphism $\varphi$ on $\left[\mathbb{F}_{2}, \mathbb{F}_{2}\right]$ that is invariant under conjugacy. Now $\left[\mathbb{F}_{2}, \mathbb{F}_{2}\right]$ is the smallest normal subgroup of $\mathbb{F}_{2}$ containing $[x, y]$. Therefore, any such homomorphism is determined by its value on $[x, y]$. In particular, if we make the analogous construction taking $g(x)=Q_{\alpha}(x, 1)$ in place of $f(y)$ and define $\psi(\alpha)=g^{\prime}(1)$, we have $\psi=-\varphi$ as we can see by evaluating on $[x, y]$. 


\section{$3 \quad$ Further criteria}

So far we have one criterion for an element in $\left[\mathbb{F}_{2}, \mathbb{F}_{2}\right]$ to be a product of two squares, namely, if $g$ is a product of two squares then $\varphi(g)$ is even. If $\varphi(g)=0$ (which implies $\psi(g)=0$ ), there are other criteria. These are obtained by constructing homomorphisms $\varphi_{2}$ and $\psi_{2}$ on appropriate subgroups of $G=$ $\left[\mathbb{F}_{2}, \mathbb{F}_{2}\right]$ which are even on elements that are products of two squares.

We first need some lemmas. Let $G_{1}$ denote the kernel of $\varphi$. Since $\varphi$ is conjugacy invariant, $G_{1}$ is a normal subgroup of $\mathbb{F}_{2}$. Let $H_{1}=\operatorname{ker}(\psi)=G_{1}$.

Lemma 3.1 Suppose $\varphi(g)=0$ and $g=a b$ with $a$ and $b$ conjugate. Then $a, b \in G_{1}$.

Proof As $\varphi$ is conjugacy invariant, $0=\varphi(g)=2 \varphi(a)$.

We need an elementary property of derivatives of polynomials.

Lemma 3.2 Let $f(t)$ be a Laurent polynomial with integer coefficients. Then $f^{k}(t)$ is divisible by $k$ !.

Proof The $k$ th derivative of $t^{n}$ is divisible by $n(n-1) \cdots(n-k+1)$ which in turn is divisible by $k !$. The result follows.

Now we can define two homomorphisms $\varphi_{2}$ and $\psi_{2}$ from $H_{1}=G_{1}$ to $\mathbb{Z}$ by $\varphi_{2}(g)=f_{\alpha}^{\prime \prime}(1) / 2$ and $\psi_{2}=g_{\alpha}^{\prime \prime}(1) / 2$ with $\alpha$ as before. The proof that these are well defined and conjugacy invariant is exactly as in the previous section.

Continuing in this manner, we let $G_{2}=\operatorname{ker}\left(\varphi_{2}\right)$ and $H_{2}=\operatorname{ker}\left(\psi_{2}\right)$. We inductively define groups $H_{k}$ and $G_{k}$ and homomorphisms $\varphi_{k}: G_{k-1} \rightarrow \mathbb{Z}$ and $\psi_{k}: H_{k-1} \rightarrow \mathbb{Z}$. Namely, let $\varphi_{k}(\alpha)=f_{\alpha}^{(k)}(1) / k$ ! and $\psi_{k}(\alpha)=g_{\alpha}^{(k)}(1) / k$ ! and define $G_{k}=\operatorname{ker}\left(\varphi_{k}\right)$ and $H_{k}=\operatorname{ker}\left(\psi_{k}\right)$.

As in the previous section, we deduce the following properties of the homomorphisms $\varphi_{k}$ and $\psi_{k}$.

Lemma 3.3 The homomorphisms $\varphi_{k}$ and $\psi_{k}$ are invariant under the action of $\mathbb{F}_{2}$ by conjugation.

Lemma 3.4 If $g \in G_{k}$ (respectively $g \in H_{k}$ ) is a product of two squares, then $\varphi_{k}(g)$ (respectively $\psi_{k}$ ) is even. 
Thus, we have infinitely many obstructions to an element being the product of two squares. More precisely, let $g \in\left[\mathbb{F}_{2}, \mathbb{F}_{2}\right]$ be an element. We evaluate $\varphi(g)=-\psi(g)$. There are three possibilities: $\varphi(g)=0, \varphi(g)$ is even or $\varphi(g)$ is odd. In case $\varphi(g)$ is odd, we know that $g$ is not a square. If it is even but nonzero, we cannot deduce any further obstructions. In the case when $\varphi(g)=0$, we have additional homomorphisms $\varphi_{2}$ and $\psi_{2}$ which can be applied to $g$ to get an odd or even number. This process can be continued inductively.

\section{Some examples}

We have constructed in the previous section two sequences of obstructions to an element $g \in\left[\mathbb{F}_{2}, \mathbb{F}_{2}\right]$ being a product of two squares, based on the homomorphisms $\varphi_{k}$ and $\psi_{k}$. We shall show that all these are non-trivial in the sense that there are elements for which the first $k-1$ homomorphisms vanish and the $k$ th is odd.

On the other hand, in the case when $f(y)=0$ and $g(x)=0$ as polynomials, all our homomorphisms vanish. We shall construct examples where this happens.

Our examples are based on the observation that the associations $\gamma \rightarrow P_{\gamma}$ and $\gamma \rightarrow Q_{\gamma}$ are module homomorphisms over the ring of Laurent polynomials in variables $x$ and $y$, from $H_{1}(\tilde{K})$ to Laurent polynomials. Further, we have a surjection from the commutator subgroup $\left[\mathbb{F}_{2}, \mathbb{F}_{2}\right]=\pi_{1}(\tilde{K})$ to its abelianisation $H_{1}(\tilde{K})$.

Proposition 4.1 For any $k>1$, there is an element $g \in\left[\mathbb{F}_{2}, \mathbb{F}_{2}\right]$ such that $\varphi_{j}(g)=0$ for all $j<k, \varphi_{k}(g)=-1$ and $\psi_{j}(g)=0$ for all $j$.

Proof Let $g$ be an element whose image in $H_{1}(\tilde{K})$ is $\gamma=(y-1)^{k-1} \alpha$, where $\alpha$ denotes the class of $[x, y]$ in $H_{1}(\tilde{K})$. As $P_{\alpha}=-(y-1)$, and the associations $\gamma \rightarrow P_{\gamma}$ and $\gamma \rightarrow Q_{\gamma}$ are module homomorphisms, it follows that $P_{\gamma}=-(y-1)^{k}$ and $Q_{\gamma}=(y-1)^{k-1}(x-1)$.

Now $f_{\gamma}(y)=P_{\gamma}(1, y)=-(y-1)^{k}$ and $g_{\gamma}(x)=Q_{\gamma}(x, 1)=0$ and hence $\varphi_{j}(g)=0$ for all $j<k, \varphi_{k}(g)=-1$ and $\psi_{j}(g)=0$ for all $j$.

Recall that we consider succesively the homorphisms $\varphi_{k}$ (and $\psi_{k}$ ), with $\varphi_{k+1}$ defined if $\varphi_{k}$ vanishes. The first non-zero $\varphi_{k}$ gives a criterion for an element being the product of two squares. We see in the next example that there are elements in $\left[\mathbb{F}_{2}, \mathbb{F}_{2}\right]$ for which all the $\varphi_{k}$ vanish. 
Proposition 4.2 There is an element $g \in\left[\mathbb{F}_{2}, \mathbb{F}_{2}\right]$ such that $\varphi_{j}(g)=0=$ $\psi_{j}(g)$, for all $j$.

Proof We take $g$ whose image in $H_{1}(\tilde{K})$ is $\gamma=(x-1)(y-1) \alpha$. Then, using the notation of the above proposition, we see that $f_{\gamma}(y)=0$ and $g_{\gamma}(x)=0$, and hence $\varphi_{j}(g)=0=\psi_{j}(g)$, for all $j$.

\section{$5 \quad$ Factorisation and another criterion}

The above example suggests a variant of our criteria.

Proposition 5.1 Let $g \in\left[\mathbb{F}_{2}, \mathbb{F}_{2}\right]$ be an element with $\alpha$ the corresponding cycle in $H_{1}(\tilde{K})$. Suppose $(x-1)^{k}(y-1)^{l}$ divides $P_{\alpha}(x, y)$ with quotient $h(x, y)$. If $g$ is a product of two squares then $h(1,1)$ is even.

Proof We have seen that if $g$ is the product of two squares, it is the product of two conjugates $c$ and $d$. Let $A b$ denote the abelianisation map $\left[\mathbb{F}_{2}, \mathbb{F}_{2}\right] \rightarrow$ $H_{1}(\tilde{K})$. Then $A b(g)=A b(c)+A b(d)$. As $c$ and $d$ are conjugate (in $\mathbb{F}_{2}$ ), they differ by a deck transformation of $\tilde{K}$. Equivalently, $c$ and $d$ differ by the action by conjugation of the abelianisation $\mathbb{F}_{2} /\left[\mathbb{F}_{2}, \mathbb{F}_{2}\right]=\mathbb{Z}^{2}$ on the commutator subgroup $\left[\mathbb{F}_{2}, \mathbb{F}_{2}\right]$. This action has been identified with multiplication by Laurent polynomials.

Hence, for some integers $m, n \in \mathbb{Z}$, we have $A b(d)=x^{m} y^{n} A b(c)$. It follows that $A b(g)=\left(x^{m} y^{n}+1\right) A b(c)$ and hence $\left(1+x^{m} y^{n}\right)$ divides $P_{\alpha}$.

Now the ring of Laurent polynomials over $\mathbb{Z}$ in $x$ and $y$ is a unique factorisation domain, and $x-1$ and $y-1$ are prime elements. Further, they do not divide $E(x, y)=\left(1+x^{m} y^{m}\right)$ as $E(1,1) \neq 0$. Thus, $E(x, y)$ divides $h(x, y)$. As $E(1,1)=2, h(1,1)$ is even.

Acknowledgements I would like to thank S. Gadgil, S. P. Inamdar and B. Sury for their advice and encouragement, which also enabled me to refine what I had first proved. I thank the referee for several helpful comments. 


\section{References}

[1] M Akhavan-Malayeri, Powers of commutators as products of squares, Int. J. Math. Math. Sci. 31 (2002) 635-637 MathReview

[2] R C Lyndon, MF Newman, Commutators as products of squares, Proc. Amer. Math. Soc. 39 (1973) 267-272 MathReview

Stat-Math Unit, Indian Statistical Institute

Bangalore, India

Email: bmat0212@isibang.ac.in

Received: 5 November 2003 Revised: 25 March 2004 